УДК 536.24

\title{
ОСОБЕННОСТИ ОПТИМИЗАЦИОННОЙ ЗАДАЧИ НА ПОДБОР МАТЕРИАЛОВ ДЛЯ МИНИМИЗАЦИИ ПОТЕРИ ТЕПЛА ЧЕРЕЗ ПЛОСКУЮ СТЕНКУ
}

\author{
Сабденов Каныш Оракбаевич1, \\ sabdenovko@yandex.kz \\ Ерзада Майра ${ }^{1}$, \\ mayira76@yahoo.co.jp \\ 1 Евразийский национальный университет им. Л.Н. Гумилева, \\ Казахстан, 010008, г. Астана (Нур-Султан), ул. Сатпаева, 2.
}

\begin{abstract}
Актуальность. Хранение тепла и его эфффективное использование связано с подбором материалов для тепловой изоляции стенок. Такие материалы представлены широким спектром теплофизических свойств и стоимости на рынке. Тогда возникает задача оптимизации, ее решение должно обеспечивать наименьшие потери тепла через стенку при ограниченном выборе материалов с заданными коэфффициентами теплопроводности. Вместе с тем при решении оптимизационной задачи могут возникнуть сложности в оценке правильности полученных результатов. Поэтому этот вопрос нуждается в детальном обсуждении.

Цель: математическое моделирование стационарных режимов переноса тепла, формулировка минимаксной задачи о потере тепла через стенку, построение области решения минимаксной задачи, анализ полученных результатов и фрормулировка выводов. объект: стенка, теплоизоляционные материалы, потоки тепла, условия минимальности, оптимальные решения. Методы: решение минимаксной задачи с применением аналитических методов.

Результаты. Сфрормулирована простая минимаксная задача: дана двухслойная плоская стенка с произвольными коэфффициентами теплопроводности и фиксированными толщинами. На правой и левой границах стенки задана постоянная и различная температура. Также задан максимальный тепловой поток через стенку и область возможных значений коэффрициентов теплопроводности (т. е. возможные материалы) для каждого слоя стенки. Требуется из этой области найти коэфрфициенты теплопроводности, обеспечивающие тепловой поток ниже заданного максимального значения. На этом примере показано, что решение поставленной минимаксной задачи может приводить к неверному результату: можно получить или не весь спектр допустимых решений, или задача может не иметь решения. Это означает необходимость строгого отношения к формулировке и методу решения оптимизационных задач для процессов переноса тепла.
\end{abstract}

\section{Ключевые слова:}

Хранение тепла, стенка, теплоизоляционный материал, минимаксная задача, коэффициенты теплопроводности, потери тепла.

\section{Введение}

Решение проблемы снижения потока тепла через стенку связано со многими техническими устройствами и технологиями, например,

- с методом хранения тепла и холода в подземных водоносных слоях ATES (Aquifer Thermal Energy Storage) [1-4];

- при использовании геотермальной энергии [5-9];

- при транспорте тепла наземными и подземными трубопроводами [10-14];

- в системах теплозащиты [15-18].

Вопрос подбора теплоизоляционного материала с нужным коэффициентом теплопроводности и его толщины можно решить прямым расчетом тепловой задачи и подбирая нужные параметры [9-10, 14], или формулировкой оптимизационных задач $[11,12]$. Оптимизационные задачи в ряде случаев дают удовлетворительные результаты не только при решении тепловых задач $[11,12,19,20]$, но и во многих других приложениях экономики, техники и технологии $[21,22]$. Однако минимаксный подход может привести к очень неожиданным результатам, и этому вопросу посвящена настоящая работа.

Для уменьшения потерь тепла через поверхности применяются однослойные и многослойные теплоизоляционные материалы. Чем меныше коэффициент теплопроводности материала и болыше толщина теплоизоляционного мате- риала, тем меныше поток тепла $j$ через наружные поверхности. При заданном ограничении на свойства материала и его толщину оптимизационная задача решается элементарно просто. Но если поверхность покрывается двумя и более различными материалами, то оптимизационная задача может привести к неожиданным результатам. Ниже это показано на простом примере.

\section{Формулировка и решение минимаксной задачи}

Задача ставится следующим образом: плоская стена составлена из двух разных материалов (1 и 2) с толщинами $a$ и $b$, их коэффициенты теплопроводности обозначены соответственно $\lambda_{1}$ и $\lambda_{2}$ (рис. 1). С левой стороны стенки поддерживается температура $T_{\text {in, }}$

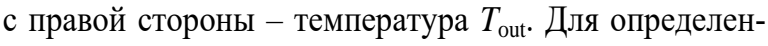
ности примем $T_{\text {in }}>T_{\text {out }}$, тогда поток тепла имеет положительный знак. В задаче дан максимальной поток тепла $j_{\max }>0$, требуется из заданной области допустимых значений $\lambda_{1} \in\left[\Lambda_{1, \min } ; \Lambda_{1, \max }\right] \cup \lambda_{2} \in\left[\Lambda_{2, \min } ; \Lambda_{2, \max }\right]$ найти все возможные коэффициенты $\lambda_{1}$ и $\lambda_{2}$, отвечающие условию $j \leq j_{\max }$.

Обозначим температуры в первом и втором материале как $T_{1}$ и $T_{2}$. В теплотехнике показано [18], что если стационарный процесс переноса тепла в плоской стенке протекает только в одном направлении, то 
распределение температуры дается линейными функциями. По рис. 1 перенос происходит только в направлении координаты $x$. Поэтому указанные функций следующие:

$$
T_{1}(x)=k_{1} x+c_{1} ; \quad T_{2}(x)=k_{2} x+c_{2} .
$$

Здесь $k_{1}, c_{1}, k_{2}, c_{2}$ - подлежащие определению неизвестные коэффициенты, они находятся из граничных условий. Опуская простые расчеты с использованием (1), приведем формулу для коэффициента $k_{1}$ :

$$
k_{1}=-\lambda_{2} \frac{T_{\text {in }}-T_{\text {out }}}{\lambda_{2} a+\lambda_{1} b} .
$$

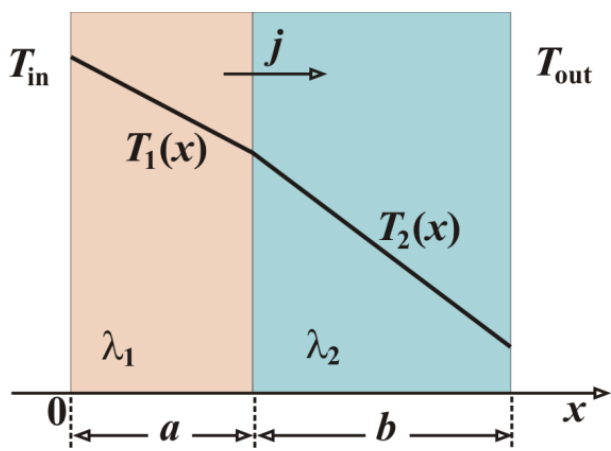

Pис. 1. Схема двуслойной стенки и принятые обозначения

Fig. 1. Scheme of the two-layer wall and the accepted notation

По условию задачи необходимо определить, для каких материалов выполняется неравенство $j \leq j_{\max }$. Чтобы написать его в виде уравнения, возьмем определение

$$
j=-\lambda_{1} \frac{d T_{1}}{d x}=-\lambda_{1} k_{1} .
$$

Тогда с его использованием условие на тепловой поток можно записать в виде

$$
-\lambda_{1} k_{1} \leq j_{\max }
$$

Подставим сюда значение $k_{1}$ из формулы (2), тогда получим:

$$
\lambda_{1} \lambda_{2} \frac{T_{\text {in }}-T_{\text {out }}}{\lambda_{2} a+\lambda_{1} b} \leq j_{\max } .
$$

Здесь могут меняться только коэффициенты теплопроводности $\lambda_{1}$ и $\lambda_{2}$, остальные параметры фиксированы. Тогда это неравенство можно рассматривать как условие, накладываемое на функцию $\lambda_{1}\left(\lambda_{2}\right)$, после простых преобразований получим следующую форму записи неравенства:

$$
\lambda_{1} \leq \frac{\lambda_{2} a}{\lambda_{2} \frac{T_{\text {in }}-T_{\text {out }}}{j_{\text {max }}}-b} .
$$

Коэффициент теплопроводности по его физическому смыслу может быть только положительным числом, поэтому должно быть

$$
\lambda_{2} \frac{T_{\text {in }}-T_{\text {out }}}{j_{\max }} \geq b
$$

или

$$
\lambda_{2} \geq b \frac{j_{\max }}{T_{\text {in }}-T_{\text {out }}} .
$$

Это первый важный результат. Следующим шагом найдем асимптоты, если идти по переменной $\lambda_{2}$ c верхней стороны:

$$
\lambda_{2} \rightarrow \frac{j_{\max } b}{T_{\text {in }}-T_{\text {out }}},
$$

то коэффициент $\lambda_{1} \rightarrow \infty$. Таким образом найдена 1 -я (вертикальная) асимптота. Вторая асимптота находится устремлением $\lambda_{2} \rightarrow \infty$, в этом случае

$$
\lambda_{1} \rightarrow \frac{j_{\text {max }} a}{T_{\text {in }}-T_{\text {out }}} .
$$

Это 2-я горизонтальная асимптота. Теперь если использовать неравенство (4) и найденные асимптоты, то на графике можем увидеть фигуру, определяемую условием (3) (рис. 2).

На этом рисунке решение задачи представляется закрашенной областью. График функции $\lambda_{1}\left(\lambda_{2}\right)$ построен по формуле

$$
\lambda_{1}=\frac{\lambda_{2} a}{\lambda_{2} \frac{T_{\mathrm{in}}-T_{\mathrm{out}}}{j_{\max }}-b} .
$$

Чтобы поток тепла был меньше указанного значения $j_{\max }$, коэффициенты теплопроводности не должны быть меньше приведенных ниже минимальных значе-

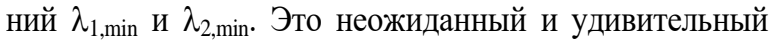
результат, минимальное значение коэффициента теплопроводности для первого материала равно

$$
\lambda_{1, \min }=\frac{j_{\max } a}{T_{\text {in }}-T_{\text {out }}} .
$$

Минимальное значение коэффициента теплопроводности для второго материала равно

$$
\lambda_{2, \min }=\frac{j_{\max } b}{T_{\text {in }}-T_{\text {out }}} .
$$

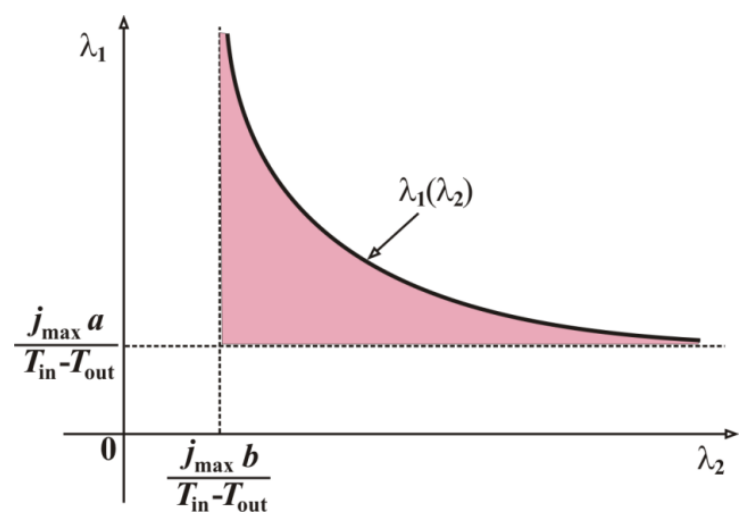

Pис. 2. Качественное представление решения задачи: область, ограничиваемая графиком функции $\lambda_{1}\left(\lambda_{2}\right)$ и двумя асимптотами

Fig. 2. Qualitative representation of the solution to the problem: the graph of function $\lambda_{1}\left(\lambda_{2}\right)$ and two asymptotes are the boundary of the solution domain 
Из полученных результатов следуют два вывода: первый - опирающееся на интуицию широко распространенное мнение, что «для обеспечения минимальности потока тепла всегда должны быть минимальными коэфициенты теплопроводности», не всегда верно; второй - заданная область допустимых значений $\lambda_{1} \in\left[\Lambda_{1, \min } ; \Lambda_{1, \max }\right] \cup \lambda_{2} \in\left[\Lambda_{2, \min } ; \Lambda_{2, \max }\right]$ может не иметь пересечений с областью на рис. 2 и тогда оптимизационная задача не имеет решения.

Полученные в настоящей работе результаты не зависят от того, на какой внешней стороне стенки выше температура. Если принять $T_{\text {in }}<T_{\text {out, }}$ то необходимо поменять знак теплового потока $j_{\max }$ на отрицательный.

Итак, действующий поток тепла при произвольных значениях коэффициентов $\lambda_{1}$ и $\lambda_{2}$ находится по формуле

$$
j=\lambda_{1} \lambda_{2} \frac{T_{\text {in }}-T_{\text {out }}}{\lambda_{2} a+\lambda_{1} b} .
$$

Посмотрим на изменение этого потока при движении по произвольно взятой кривой

$$
\lambda_{1}=0,1+1,3 \lambda_{2}
$$

на плоскости переменных $\left(\lambda_{1}, \lambda_{2}\right)$ при заданном $j_{\max }$. На рис. 3 приведены результаты расчета при следующих параметрах:

$$
\begin{gathered}
j_{\text {max }}=130 \mathrm{BT} / \mathrm{M}^{2} ; T_{\text {in }}=120{ }^{\circ} \mathrm{C} ; T_{\text {out }}=-20{ }^{\circ} \mathrm{C} ; \\
a=0,15 \mathrm{~m} ; b=0,32 \mathrm{M} .
\end{gathered}
$$

Минимальные значения коэффициентов теплопроводности $\lambda_{1, \min }=0,75 \mathrm{BT} /(\mathrm{M} \cdot \mathrm{K}), \lambda_{2, \min }=0,297 \mathrm{BT} /(\mathrm{M} \cdot \mathrm{K})$. Граничная кривая 2 на рис. 3 построена для того, чтобы видеть область, отвечающую условию минимальности теплового потока. При движении по произвольной кривой 2 слева направо происходит переход через граничную кривую. Вертикальная штриховая линия указывает на точку перехода $\lambda_{2}=0,357 \mathrm{BT} /(\mathrm{м} \cdot \mathrm{K})$ и соответствующую ей $\lambda_{1}=0,601 \mathrm{BT} /(\mathrm{M} \cdot \mathrm{K})$. Двигаясь по ней выше, определяем, что эти два значения коэффициента теплопроводности дают верхнюю предельную величину $j_{\max }=130 \mathrm{BT} / \mathrm{m}^{2}$. Таким образом, граница по уравнению (5) точно определяет предельный поток $j_{\max }$, и все лежащие ниже границы 1 точки на рис. 3 приводят к потоку $j<j_{\max }$.

Но почему-то точки на плоскости $\left(\lambda_{1}, \lambda_{2}\right)$, лежащие ниже горизонтальной и слева вертикальной асимптоты, «выпали» из решения. Если посмотреть на формулы для температуры в 1 и 2 областях:

$$
\begin{gathered}
T_{1}=T_{\text {in }}-\lambda_{2} \frac{T_{\text {in }}-T_{\text {out }}}{\lambda_{2} a+\lambda_{1} b} x, \\
T_{2}=T_{\text {out }}+\lambda_{1} \frac{T_{\text {in }}-T_{\text {out }}}{\lambda_{2} a+\lambda_{1} b}(a+b-x),
\end{gathered}
$$

то отсюда и из формулы (6) видно, что решение для распределения температуры существует при любых коэффициентах $\lambda_{1}$ и $\lambda_{2}$, на тепловой поток $j$ нет никаких ограничений.

Рассмотрим сначала в формулах (8) предельные случаи $\lambda_{1} \rightarrow 0$ и $\lambda_{2} \rightarrow 0$, но так, чтобы всегда $\lambda_{1} \neq 0$ и $\lambda_{2} \neq 0$.Одновременно такие пределы трудно выполнить, поэтому необходимо в первом уравнении сначала устремить $\lambda_{1} \rightarrow 0$, а во втором уравнении $\lambda_{2} \rightarrow 0$. Тогда из (8) следуют распределения

$$
\begin{gathered}
T_{1}=T_{\text {in }}-\frac{T_{\text {in }}-T_{\text {out }}}{a} x, \\
T_{2}=T_{\text {out }}+\frac{T_{\text {in }}-T_{\text {out }}}{b}(a+b-x) .
\end{gathered}
$$

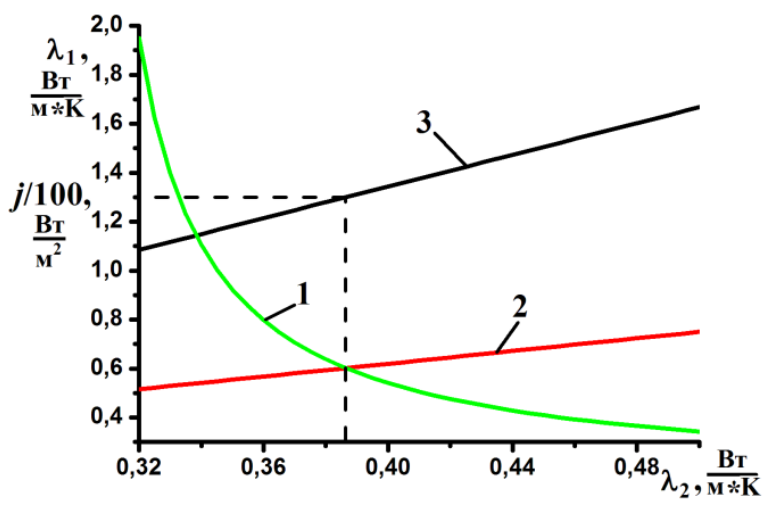

Pис. 3. Результаты расчета теплового потока (кривая 3) по уравнению (6) и коэффичиента теплопроводности $\lambda_{1}: 1$ - граничная кривая по формуле (5); 2 - произвольно взятая кривая по формуле (7)

Fig. 3. Results of calculating the heat flux (curve 3) according to equation (6) and the thermal conductivity coefficient $\lambda_{l}: 1$ - boundary curve according to formula (5); 2 - arbitrary curve taken by the formula (7)

Эти распределения температуры не зависят от $\lambda_{1}$ и $\lambda_{2}$, а только от координаты, толщин теплоизоляции, граничных температур. В указанных пределах тепловой поток через стенку становится сколь угодно малым. Теперь вместо коэффициентов теплопроводности подставим в (8) найденные выше их предельные значения $\lambda_{1, \min }$ и $\lambda_{2, \min }$, им соответствует угловая точка на рис. 2 , где поток тепла минимален. Тогда получим

$$
\begin{gathered}
T_{1}=T_{\text {in }}-\frac{T_{\text {in }}-T_{\text {out }}}{2 a} x, \\
T_{2}=T_{\text {out }}+\frac{T_{\text {in }}-T_{\text {out }}}{2 b}(a+b-x) .
\end{gathered}
$$

Здесь снова обнаруживаем аналогичную, как в предыдущем случае (9), ситуацию зависимости решений для температуры только от координат и граничных значений $T_{\text {in }}, T_{\text {out }}$ Соответствующий распределениям (10) поток тепла равен

$$
j=\frac{1}{2} j_{\max }
$$

Таким образом, в угловой точке $\left(\lambda_{1, \min }, \lambda_{2, \min }\right)$ поток тепла в два раза ниже заданной предельной величины.

\section{Заключение}

На примере простой задачи о переносе тепла через составленную из двух различных материалов плоскую стенку показано, что оптимизационная задача на 
их подбор по величине коэффициентов теплопроводности может привести к неполному решению. Поэтому при проектировании теплоизоляционных покрытий на основе данных, полученных из решения минимаксных задач, необходимо проводить дополнительный анализ. Такой подход не является самым

\section{СПИСОК ЛИТЕРАТУРЫ}

1. Schuppler S., Fleuchaus P., Blum Ph. Techno-economic and environmental analysis of an Aquifer Thermal Energy Storage (ATES) in Germany // Geothermal Energy. - 2019. - V. 7. № 11. - P. 1-24.

2. Aquifer thermal energy storage: theoretical and operational analysis / J.S. Dickinson, N. Buik, M.C. Matthews, A. Snijders // Geotechnique. - 2009. - V. 59. - № 3. - P. 249-260.

3. A review on system performance studies of aquifer thermal energy storage / Liuhua Gao, Jun Zhao, Qingsong An, Junyao Wang, Xueling Liu // Energy Procedia: 9th International Conference on Applied Energy, ICAE2017. - Cardiff, UK, 21-24 August 2017. № 142. - P. 3537-3545.

4. Possemiers M., Huysmans M., Batelaan O. Influence of aquifer thermal energy storage on groundwater quality: a review illustrated by seven case studies from Belgium // Journal of Hydrology: Regional Studies. - 2014. - № 2. - P. 20-34.

5. Hähnlein S., Bayer P., Blum P. International legal status of the use of shallow geothermal energy // Renewable and Sustainable Energy Reviews. - 2010. - V. 14. - Iss. 9. - P. 2611-2625.

6. Understanding the Chena Hot flopë Springs, Alaska, geothermal system using temperature and pressure data / K. Erkan, G. Holdmann, W. Benoit, D. Blackwell // Geothermics. - 2008. V. 37. - № 6. - P. 565-585.

7. Miglani S., Orehounig K., Carmeliet J. A methodology to calculate long-term shallow geothermal energy potential for an urban neighbourhood // Energy and Buildings. - 2018. - V. 159. P. 462-473.

8. Fridleifsson I.B. Geothermal energy for the benefit of the people // Renewable and Sustainable Energy Reviews. - 2001. - V. 5. Iss. 3. - P. 299-312

9. Максимов В.И., Салум А. Особенности использования геотермальной энергии с применением теплонасосных установок в условиях низких температур // Известия ТПУ. Инжиниринг георесурсов. - 2019. - Т. 330. - № 2. - С. 115-123.

10. Половников В.Ю. Численный анализ влияния нестационарности процессов теплопереноса в зонах размещения подземных теплопроводов на их тепловые режимы и тепловые потери // Известия ТПУ. Инжиниринг георесурсов. - 2018. - Т. 329. № 10. - С. 76-84. трудоемким, поскольку прямой путь, заключающийся в решении задач теплопереноса и перебора всех возможных значений коэффициентов теплопроводности из заданной области значений, может привести к еще большим затратам времени и средств.

11. Сабденов К.О., Байтасов Т.М. Оптимальное (энергоэффективное) теплоснабжение здания в системе центрального отопления // Известия ТПУ. Инжиниринг георесурсов. - 2015. T. 326. - №. 8. - Р. 53-60.

12. Сабденов К.О. Минимаксный анализ экономической и энергетической эффективностей трубопроводов теплоснабжения // Инженерно-физический журнал. - 2016. - Т. 89. - № 6. C. $1421-1429$.

13. EnergyPlus. Engineering Reference / Ed. by E. Orlando. - 2013. URL: http://energy.gov/eere/office-energy-efficiency-renewableenergy (дата обращения: 14.09.2014).

14. Половников В.Ю., Губина Е.В. Тепломассоперенос в увлажненной тепловой изоляции теплопроводов, работающих в условиях затопления // Инженерно-физический журнал. 2014. - T. 87. - № 5 - C. 1106-1112.

15. Основы теплопередачи в авиационной и ракетно-космической технике / В.С. Авдуевский, Б.М. Галицейский, Г.А. Глебов и др. - М.: Машиностроение, 1975. - 528 с.

16. Половников В. Ю. Кондуктивный теплоперенос в слое тонкопленочной тепловой изоляции // Известия ТПУ. Инжиниринг георесурсов. - 2019. - Т. 330. - № 5. - С. 189-197.

17. Салахутдинов Г.М. Тепловая защита в космической технике // Новое в жизни, науке, технике: космонавтика, астрономия. М.: Знание, 1982. -64 c.

18. Зингер Н.М. Гидравлические и тепловые режимы теплофикационных сетей. 2-е изд. - М.: Энергоатомиздат, 1986. - 320 с.

19. Сабденов К.О., Байтасов Т.М., Ерзада М. Оптимальное регулирование теплоснабжением здания. Часть 1. Формулировка задачи и основные формулы // Инженерно-физический журнал. - 2014. - Т. 87. - № 4. - С. 814-821.

20. Сабденов К.О., Байтасов Т.М., Ерзада М. Оптимальное регулирование теплоснабжением здания. Часть 2. Анализ и результаты // Инженерно-физический журнал. - 2014. - Т. 87. № 4. - C. 822-828.

21. Алексеев В.М., Галлеев Э.М., Тихомиров В.М. Сборник задач по теории оптимизации. Теория, примеры, задачи. - М.: Физматлит, 2005. -288 c.

22. Габасов Р., Кириллова Ф.М. Методы оптимизации. - Минск: Изд-во БГУ, 1980. -472 с.

Поступила 03.06.2020 г.

\section{Информация об авторах}

Сабденов К.О., доктор физико-математических наук, профессор, Евразийский национальный университет им. Л.Н. Гумилева.

Epзада M., доктор технических наук, доцент, Евразийский национальный университет им. Л.Н. Гумилева. 
UDC 536.24

\title{
FEATURES OF OPTIMIZATION PROBLEM FOR SELECTING MATERIALS TO MINIMIZE HEAT LOSS THROUGH THE WALL
}

\author{
Kanysh O. Sabdenov1, \\ sabdenovko@yandex.kz \\ Maira Erzada 1 , \\ mayira76@yahoo.co.jp \\ 1 L.N. Gumilev Eurasian National University, \\ 2, Satpaev street, Astana (Nur-Sultan), 010008, Kazakhstan.
}

The relevance. Heat storage and its effective use is associated with the selection of materials for thermal insulation of the walls. Such materials are represented by a wide range of thermophysical properties and cost in the market. Then the optimization problem arises, its solution should provide the smallest heat loss through the wall with a limited choice of materials with the given thermal conductivity coefficients. However, when solving the optimization problem, difficulties may arise in assessing the correctness of the results obtained. Therefore, this issue needs a detailed discussion.

The main aim of the research is mathematical modeling of stationary modes of heat transfer, formulation of the minimax problem of heat loss through the wall, construction of the solution area of the minimax problem, the analysis of the results and conclusions.

Object: wall, heat-insulating materials, heat fluxes, minimalist conditions, optimal solutions.

Methods: solving a minimax problem using analytical methods.

Results. The authors have stated the simple minimax problem: a two-layer flat wall is given with arbitrary heat conductivity coefficients and fixed thicknesses. On the right and left borders of the wall, a constant and different temperature is set. The maximum heat flux through the wall and the range of possible values of the thermal conductivity coefficients (i. e., possible materials) for each wall layer are also specified. It is required to find such heat conductivity coefficients from this region that ensure the heat flux below a given maximum value. This example shows that the solution of the minimax problem posed can lead to an incorrect result: either the whole range of feasible solutions can be obtained, or the problem may not have a solution. This means the need for a strict attitude to the formulation and method of solving optimization problems for heat transfer.

\section{Key words:}

Heat storage, wall, heat-insulating material, minimax problem, thermal conductivity coefficients, heat loss.

\section{REFERENCES}

1. Schuppler S., Fleuchaus P., Blum Ph. Techno-economic and environmental analysis of an Aquifer Thermal Energy Storage (ATES) in Germany. Geothermal Energy, 2019, vol. 7, no. 11, pp. 1-24.

2. Dickinson J.S., Buik N., Matthews M.C., Snijders A. Aquifer thermal energy storage: theoretical and operational analysis. Geotechnique, 2009, vol. 59, no. 3, pp. 249-260.

3. Liuhua Gao, Jun Zhao, Qingsong An, Junyao Wang, Xueling Liu. A review on system performance studies of aquifer thermal energy storage. Energy Procedia: $9^{\text {th }}$ International Conference on Applied Energy, ICAE2017. Cardiff, UK, 21-24 August 2017. No. 142. pp. 3537-3545.

4. Possemiers M., Huysmans M., Batelaan O. Influence of aquifer thermal energy storage on groundwater quality: a review illustrated by seven case studies from Belgium. Journal of Hydrology: Regional Studies, 2014, no. 2, pp. 20-34.

5. Hähnlein S., Bayer P., Blum P. International legal status of the use of shallow geothermal energy. Renewable and Sustainable Energy Reviews, 2010, vol. 14, Iss. 9, pp. 2611-2625.

6. Erkan K., Holdmann G., Benoit W., Blackwell D. Understanding the Chena Hot flopë Springs, Alaska, geothermal system using temperature and pressure data. Geothermics, 2008, vol. 37, no. 6, pp. 565-585.

7. Miglani S., Orehounig K., Carmeliet J. A methodology to calculate long-term shallow geothermal energy potential for an urban neighbourhood. Energy and Buildings, 2018, vol. 159, pp. 462-473.

8. Fridleifsson I.B. Geothermal energy for the benefit of the people. Renewable and Sustainable Energy Reviews, 2001, vol. 5, Iss. 3, pp. 299-312.

9. Maksimov V.I., Saloum A. Mathematical modeling of heat transfer by operation of geothermal heat pumps. Bulletin of the Tomsk Polytechnic University. Geo Assets Engineering, 2019, vol. 330, no. 4, pp. 126-135. In Rus.
10. Polovnikov V.Yu. Numerical analysis of nonstationary heat transfer influence in the zones of underground thermal pipelines on their thermal regimes and heat losses. Bulletin of the Tomsk Polytechnic University. Geo Asset Engineering, 2018, vol. 329, no. 10, pp. 76-84. In Rus.

11. Sabdenov K.O., Baitasov T.M. Optimal (energy efficient) heat supply to buildings in central heating system. Bulletin of the Tomsk Polytechnic University. Geo Assets Engineering, 2015, vol. 326, no. 8, pp. 53-60. In Rus.

12. Sabdenov K.O. Minimax analysis of economic and energy efficiencies of heat-supply pipelines. Journal of Engineering Physics and Thermophysics, 2016, vol. 89, no. 6, pp. 1401-1409.

13. EnergyPlus. Engineering Reference. Ed. by E. Orlando. 2013. Available at: http://energy.gov/eere/ office-energy-efficiencyrenewable-energy (accessed 14 September 2014).

14. Polovnikov V.Yu., Gubina E.V. Heat and mass transfer in a wetted thermal insulation of hot water pipes operating under flooding conditions. Journal of Engineering Physics and Thermophysics, 2014, vol. 87, Iss. 5, pp. 1151-1158. In Rus.

15. Avduevsky V.S., Galitseysky B.M., Glebov G.A. Osnovy teploperedachi $v$ avizionnoy $i$ raketno-kosmicheskoy tekhnike [Fundamentals of heat transfer in aviation and rocket and space technology]. Moscow, Engineering Publ., 1975. 528 p.

16. Polovnikov V.Yu. Conductive heat transfer in layer of thin-film thermal insulation. Bulletin of the Tomsk Polytechnic University. Geo Asset Engineering, 2019, vol. 330, no. 5, pp. 189-197. In Rus.

17. Salakhutdinov G.M. Teplovaya zashchita v kosmicheskoy tekhnike [Thermal protection in space technology]. Novoe v zhizne, nauke, tekhnike: kosmonavtika, astronomiya [New in Life, Science and Technology: Cosmonautics and Astronomy]. Moscow, Znanie Publ., 1982. $62 \mathrm{p}$.

18. Singer N.M. Gidravlicheskiye $i$ teplovyye rezhimy teplofikatsionnykh setey [Hydraulic and thermal modes of heating networks]. $2^{\text {nd }}$ ed. Moscow, Energoatomizdat Publ., 1986. 320 p. 
19. Sabdenov K.O., Baitasov T.M., Erzada M. Optimum control of heat supply of a building. 1. Formulation of the problem and basic formulas. Journal of Engineering Physics and Thermophysics, 2014, vol. 87, Iss. 4, pp. 839-847.

20. Sabdenov K.O., Baitasov T.M., Erzada M. Optimum control of heat supply of a building. 2. Analysis and results. Journal of Engineering Physics and Thermophysics, 2014, vol. 87, Iss. 4, pp. $848-854$
21. Alekseev V.M., Galleev E.M., Tikhomirov V.M. Sbornik zadach po teorii optimizatsii. Teoriya, primery, zadachi [A collection of problems in optimization theory. Theory, examples and tasks]. Moscow, Fizmatlit Publ., 2005. 288 p.

22. Gabasov R., Kirillova F. M. Metody optimizatsii [Optimization methods]. Minsk, Belarusian State University Publ. house, 1980. $472 \mathrm{p}$.

Received: 3 June 2020.

\section{Information about the authors}

Kanysh Sabdenov, doctor of physic and mathematic sciences, professor, L.N. Gumilev Eurasian National University. Erzada Maira, doctor of engineering sciences, ass. professor, L.N. Gumilev Eurasian National University. 